\title{
Different Methylation of CpG-SNPs in Behcet's Disease
}

\author{
Yang Huang $(\mathbb{D}$, Handan Tan, Qingfeng Cao $(\mathbb{D}$, Gangxiang Yuan $(\mathbb{D}$, \\ Guannan Su $\mathbb{D}$, and Peizeng Yang \\ The First Affiliated Hospital of Chongqing Medical University, Chongqing Key Laboratory of Ophthalmology \\ and Chongqing Eye Institute, Chongqing, China \\ Correspondence should be addressed to Peizeng Yang; peizengycmu@126.com
}

Received 18 January 2019; Revised 4 May 2019; Accepted 7 May 2019; Published 16 May 2019

Academic Editor: Heide Schatten

Copyright (C) 2019 Yang Huang et al. This is an open access article distributed under the Creative Commons Attribution License, which permits unrestricted use, distribution, and reproduction in any medium, provided the original work is properly cited.

\begin{abstract}
Purpose. We recently performed an Epigenome-Wide Association Studies (EWAS) study in Behcet's disease (BD) and identified various cytosine-phosphate-guanine $(\mathrm{CpG})$ loci that were aberrantly methylated. In the current study, we wanted to investigate whether these sites contained genetic polymorphisms and whether the frequency of these polymorphisms was altered in BD. Methods. A two-stage study was performed. The first stage involved $358 \mathrm{BD}$ patients and 704 healthy controls to investigate genetic variants of 10 CpG-SNPs (rs10454134, rs176249, rs3808620, rs10176517, rs11247118, rs78016579, rs9461624, rs10492166, rs34929465, and rs6507921) using an iPLEX Gold genotyping assay and a Sequenom MassARRAY. In the second stage, an additional 172 independent $\mathrm{BD}$ patients and 330 healthy individuals are to confirm trends found in the first stage. Results. A higher frequency of both the rs10454134 AG genotypes ( $\mathrm{p}=0.008, \mathrm{OR}=1.413,95 \% \mathrm{CI}=1.094-1.826)$ and a lower GG genotype frequency $(\mathrm{p}=0.003$, $\mathrm{OR}=0.630,95 \% \mathrm{CI}=0.465-0.854)$ were found in $\mathrm{BD}$ patients compared to the controls in the first stage. However, after correcting for multiple comparisons, all associations identified in the first stage lost statistical significance. The frequencies of the other CpGSNPs investigated were not different between BD patients and controls. The second stage was designed using an additional cohort to confirm the association with CpG-SNP, rs10454134. The data failed to confirm the association between this CpG-SNP and BD. Conclusions. This study did not show an association between BD and CpG-SNPs in gene sites that were earlier shown to be aberrantly methylated.
\end{abstract}

\section{Introduction}

Behcet's disease (BD) is a chronic, relapsing, and multisystemic inflammatory disorder. Its classical clinical characteristics include oral aphthae, genital ulcers, multiform skin lesions, and recurrent uveitis with hypopyon [1]. Its etiology and pathogenesis are not yet fully understood. Generally, it is thought that BD is caused by the interaction of genetic variation and environmental factors. To date, there are numerous genes shown to be associated with $\mathrm{BD}$ [2-4]. DNA methylation is a pivotal part of the epigenome and provides highly complementary data on the regulation of genomic regions $[5,6]$. Previous study proved that epigenetic modification of some genes dynamics is involved in the pathogenesis of BD, such as cytoskeletal [7]. Furthermore, aberrant changes in DNA methylation have been shown to lead to abnormal gene expression in the pathogenesis of $\mathrm{BD}$, including IL6, IL10, SOCS1, IRF8, GATA3, and TGF- $\beta$ [8-12].
Sequence variations can change the $\mathrm{CpG}$, which may result in indifferences in DNA methylation between individuals. Cytosine-phosphate-guanine single nucleotide polymorphisms (CpG-SNPs) are point mutated CpG sites [13] and may play a role in the methylation status of this gene region. DNA methylation is an important gene silencing mechanism and may play a role in controlling pathways of inflammation [14]. CpG-SNPs in the promoter regions have been found to be associated with various disorders, including type 2 diabetes [15], breast cancer [16], coronary heart disease [17], and psychosis [18]. However, SNPs located in $\mathrm{CpG}$ sites in patients with autoimmune diseases such as BD have not been reported and were therefore the subject of the study described here. Functional CpG-SNPs with an aberrant methylation status were selected from the Epigenome-Wide Association Study (EWAS) we performed earlier [19], and genotype frequency was compared between $\mathrm{BD}$ patients and healthy controls. 
TABLE 1: Clinical features, age, and sex distribution of BD patients and healthy controls.

\begin{tabular}{lcc}
\hline Clinical Features & Total & $\%$ \\
\hline Patients with $\mathrm{BD}^{a}$ & 530 & \\
Mean age $\pm \mathrm{SD}^{\mathrm{b}}$ & $34.3 \pm 9.6$ & 82.1 \\
Male & 435 & 17.9 \\
Female & 95 & 98.1 \\
Uveitis & 520 & 100.0 \\
Oral ulcer & 530 & 54.0 \\
Genital ulcer & 286 & 74.5 \\
Skin lesion & 395 & 20.4 \\
Arthritis & 108 & 12.3 \\
Pathergy reaction & 65 & \\
Controls & 1034 & \\
Mean age \pm SD & $39.3 \pm 10.5$ & 48.9 \\
Male & 506 & 51.1 \\
Female & 528 & \\
\hline
\end{tabular}

a: $\mathrm{BD}=$ Behcet's disease,

b: $\mathrm{SD}=$ standard deviation;

\section{Materials and Methods}

2.1. Study Population. The study recruited a total of $530 \mathrm{BD}$ patients and 1034 healthy individuals (Table 1), visiting the uveitis center of the First Affiliated Hospital of Chongqing Medical University from January 2009 to September 2017. All individuals were Chinese Han and all BD patients had uveitis. BD patients and controls were matched according to race which all from Chinese Han and geography. Healthy individuals who had systemic immune diseases or other chronic diseases were excluded. Diagnosis of BD was made according to the standard International Study Group for $\mathrm{BD}$, requiring the presence of oral ulceration as well as any two of the following symptoms: genital ulceration, uveitis, multiform lesions, or a positive pathergy test [20]. The local research ethics committee of the First Affiliated Hospital of Chongqing Medical University (permit no. 2009-201008) approved the study. A written informed consent was obtained from all participants which abided by the tenets of the Declaration of Helsinki.

2.2. CpG-SNPs Screening. Target CpG-SNPs were selected according to our previous EWAS results [19] that included $60 \mathrm{BD}$ patients and 60 matched healthy controls using the Illumina Human Methylation450K platform (Illumina, San Diego, CA, USA). A series of screening criteria was used to find target sites including a CpG-SNP methylation $\mathrm{p}$ value less than 0.05 and a Beta.Difference either less than -0.14 or more than 0.14 or $\mathrm{p}$ value $<10^{-5}$. Beta values used to score the methylation level ranging from 0 (unmethylated) to totally methylated [21, 22]. In addition, the minor allele frequency (MAF) in the Chinese Han population needed to be greater than 0.05, excluding the sites not included in the Han Chinese Hap Map database (https://www.ncbi.nlm.nih.gov/ snp/). Furthermore, according to the UCSC (GRCh37/hg19) and HaploReg v4.1 (http://pubs.broadinstitute.org/mammals/haploreg/haploreg.php) databases [23, 24], the CpG-SNP sites located in potential functional regions (promoter region, enhancer region, CCCTC-binding factor (CTCF) binding region, first exon region, 5'UTR, TSS1500) [25] were selected. Finally, linkage disequilibrium (LD) data from the Han Chinese Hap Map database were also used to exclude SNPs in LD with each other. In total, $10 \mathrm{CpG-SNPs} \mathrm{were}$ selected based on these criteria and included rs10454134, rs176249, rs3808620, rs10176517, rs11247118, rs78016579, rs9461624, rs10492166, rs34929465, and rs6507921 (Table 3).

2.3. DNA Extraction and Genotyping. The two experimental groups including both $\mathrm{BD}$ individuals and healthy controls donated peripheral blood, which was applied to extract genomic DNA extraction with a QIAmp DNA Blood Mini Kit (Qiagen Inc., Valencia, CA, USA) and stored in 3.2\% sodium citrate-treated tubes at $-80^{\circ} \mathrm{C}$. The iPLEX Gold genotyping assay and Sequenom MassARRAY (Sequenom, CA, USA) were performed to identify genotype of the 10 CpG-SNPs. iPLEX reactions primers were designed by SNP Assay Design software (version 3.0) (Table 2). All procedures were performed according to manufacturer's instructions.

2.4. Statistical Analysis. The healthy controls should satisfy Hardy-Weinberg equilibrium (HWE) (p value $<0.05)$. HardyWeinberg equilibrium analysis was using chi-square $(\chi 2)$ test, while the genotype frequency was evaluated by direct counting. Fisher's exact test or $\chi 2$ test was applied to estimate the differences in the allele and genotype frequencies of all CpG-SNPs between BD patients and healthy controls. P value was carried out by SPSS (version 17.0; SPSS Inc., Chicago, IL). Multiple comparisons were performed to correct P-value by using the Bonferroni method, whereby the $p$ value was multiplied with the number of comparisons ( $\mathrm{P}$ corrected (Pc)). When $\mathrm{Pc}_{\mathrm{c}}<0.05$, it was considered to be significant.

\section{Results}

3.1. Clinical Features of the Subjects. The demographics and clinical symptoms of the BD patients and the healthy controls are displayed in Table 1 . The healthy cohort consisted of 506 men and 528 women, who were on average $39.3 \pm 10.5$ years old. The BD patients comprised of 435 men and 95 women, with an average age of $34.3 \pm 9.6$.

3.2. CpG-SNPs Selected. SNPs located in the CpG loci with a CpG-SNP methylation level with a $p$ value of $<0.05$ and a Beta.Difference either less than -0.14 or more than 0.14 and $\mathrm{MAF}>0.05$ were identified and eleven CpG-SNPs were included, according to the following criteria: (1) the MAF in the Chinese Han population was greater than 0.05 (https:// www.ncbi.nlm.nih.gov/snp/); (2) CpG-SNP sites are located in potential functional regions according to UCSC (GRCh37/hg19) and HaploReg v4.1 (http://pubs.broadinstitute.org/mammals/haploreg/haploreg.php) databases [23, 24]; (3) linkage disequilibrium (LD) data from the Han Chinese Hap Map database was considered. Three CpG-SNPs were chosen: rs78016579, rs34929465, and rs6507921. The methylation levels of CpG-SNPs were considered with any sites with a $\mathrm{p}$ value $<10^{-5}$ and the MAF of SNP in the CpG site 


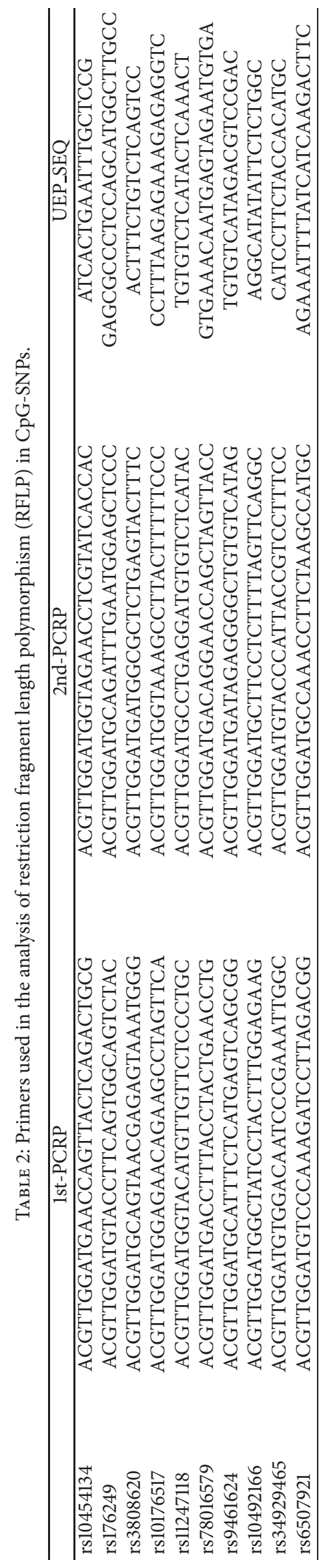




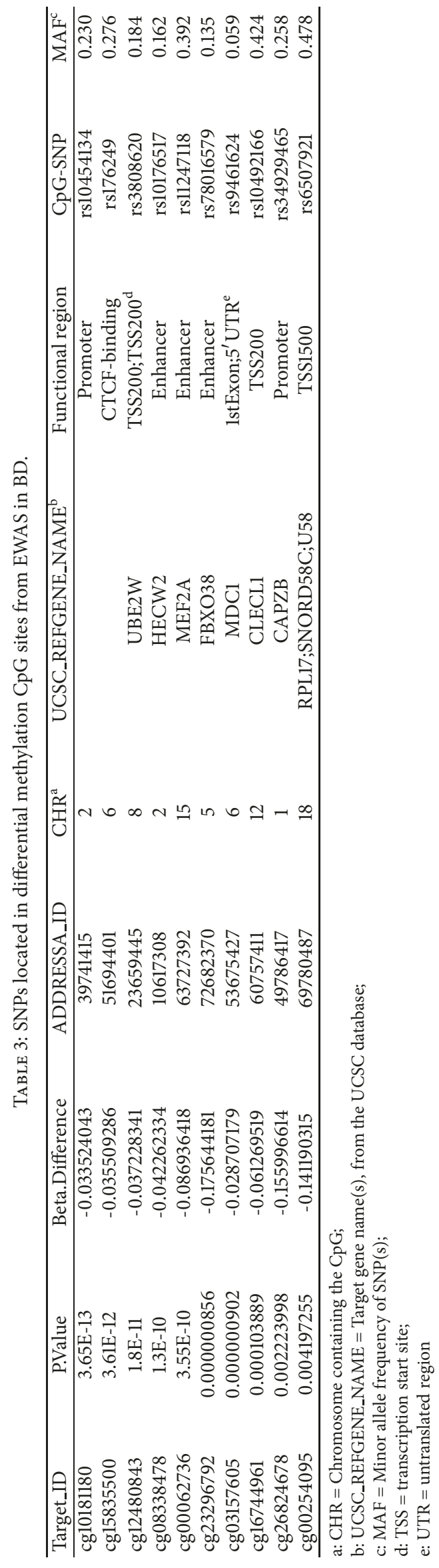


TABLE 4: Genotype and allele frequencies of CpG-SNP polymorphisms in BD and healthy controls (first stage study).

\begin{tabular}{|c|c|c|c|c|c|c|c|c|}
\hline Target_ID & CpG-SNP & Genotype & BD n (\%) & Controls n (\%) & $P$ value & Pc value & OR & $95 \% \mathrm{CI}$ \\
\hline \multirow[t]{6}{*}{$\operatorname{cg} 10181180$} & rs10454134 & total sample & 355 & 704 & & & & \\
\hline & & AA & $87(0.245)$ & $173(0.246)$ & 0.981 & NS & 0.996 & $0.741-1.340$ \\
\hline & & AG & $195(0.549)$ & $326(0.463)$ & 0.008 & NS & 1.413 & $1.094-1.826$ \\
\hline & & GG & $73(0.206)$ & $205(0.291)$ & 0.003 & NS & 0.630 & $0.465-0.854$ \\
\hline & & $\mathrm{A}$ & $369(0.520)$ & $672(0.477)$ & 0.065 & NS & 1.185 & $0.989-1.420$ \\
\hline & & G & $341(0.480)$ & $736(0.523)$ & & & 0.844 & $0.704-1.011$ \\
\hline \multirow[t]{6}{*}{ cg15835500 } & rs176249 & total sample & 357 & 702 & & & & \\
\hline & & $\mathrm{AA}$ & $13(0.036)$ & $25(0.036)$ & 0.947 & NS & 1.023 & $0.517-2.025$ \\
\hline & & AG & $119(0.333)$ & $207(0.295)$ & 0.200 & NS & 1.196 & $0.910-1.572$ \\
\hline & & GG & $225(0.630)$ & $470(0.670)$ & 0.203 & NS & 0.841 & $0.645-1.098$ \\
\hline & & $\mathrm{A}$ & $145(0.203)$ & $257(0.183)$ & 0.266 & NS & 1.137 & $0.906-1.427$ \\
\hline & & G & $569(0.797)$ & 1147 (0.817) & & & 0.879 & $0.701-1.103$ \\
\hline \multirow[t]{6}{*}{ cg12480843 } & rs3808620 & total sample & 355 & 704 & & & & \\
\hline & & GG & $339(0.955)$ & $670(0.952)$ & 0.815 & NS & 1.075 & $0.585-1.976$ \\
\hline & & CG & $16(0.045)$ & $33(0.047)$ & 0.895 & NS & 0.960 & $0.521-1.768$ \\
\hline & & $\mathrm{CC}$ & $0(0.000)$ & $1(0.001)$ & 0.477 & NS & I & I \\
\hline & & $\mathrm{G}$ & $694(0.977)$ & $1373(0.975)$ & 0.742 & NS & 1.106 & $0.608-2.012$ \\
\hline & & $\mathrm{C}$ & $16(0.023)$ & $35(0.025)$ & & & 0.904 & $0.497-1.645$ \\
\hline \multirow[t]{6}{*}{$\operatorname{cg} 08338478$} & rs10176517 & total sample & 354 & 703 & & & & \\
\hline & & CC & $190(0.537)$ & $415(0.590)$ & 0.096 & NS & 1.244 & $0.962-1.609$ \\
\hline & & $\mathrm{CT}$ & $145(0.410)$ & $253(0.360)$ & 0.115 & NS & 0.810 & $0.624-1.053$ \\
\hline & & $\mathrm{TT}$ & $19(0.054)$ & $35(0.050)$ & 0.787 & NS & 0.924 & $0.520-1.640$ \\
\hline & & $\mathrm{C}$ & $525(0.742)$ & $1083(0.770)$ & 0.144 & NS & 1.169 & $0.948-1.441$ \\
\hline & & $\mathrm{T}$ & $183(0.258)$ & $323(0.230)$ & & & 0.856 & $0.694-1.055$ \\
\hline \multirow[t]{6}{*}{$\operatorname{cg} 03157605$} & rs9461624 & total sample & 357 & 704 & & & & \\
\hline & & GG & $325(0.910)$ & $619(0.879)$ & 0.162 & NS & 0.739 & $0.484-1.130$ \\
\hline & & TG & $32(0.090)$ & $84(0.119)$ & 0.182 & NS & 1.334 & $0.873-2.040$ \\
\hline & & $\mathrm{TT}$ & $0(0.000)$ & $1(0.001)$ & 0.476 & NS & I & 1 \\
\hline & & G & $682(0.955)$ & $1322(0.939)$ & 0.122 & NS & 0.721 & $0.476-1.094$ \\
\hline & & $\mathrm{T}$ & $32(0.045)$ & $86(0.061)$ & & & 1.386 & $0.914-2.102$ \\
\hline \multirow[t]{6}{*}{ cg16744961 } & rs10492166 & total sample & 358 & 696 & & & & \\
\hline & & GG & $123(0.344)$ & $251(0.361)$ & 0.584 & NS & 0.928 & $0.710-1.212$ \\
\hline & & GA & $169(0.472)$ & $344(0.494)$ & 0.495 & NS & 0.915 & $0.709-1.181$ \\
\hline & & AA & $66(0.184)$ & $101(0.145)$ & 0.098 & NS & 1.332 & $0.947-1.871$ \\
\hline & & G & $415(0.580)$ & $846(0.608)$ & 0.212 & NS & 0.890 & $0.741-1.069$ \\
\hline & & A & $301(0.420)$ & $546(0.392)$ & & & 1.124 & $0.936-1.350$ \\
\hline \multirow[t]{6}{*}{ cg00254095 } & rs6507921 & total sample & 358 & 677 & & & & \\
\hline & & $\mathrm{CC}$ & $103(0.288)$ & $232(0.343)$ & 0.072 & NS & 1.291 & 0.977-1.705 \\
\hline & & $\mathrm{CT}$ & $181(0.506)$ & $308(0.455)$ & 0.121 & NS & 0.816 & $0.632-1.055$ \\
\hline & & $\mathrm{TT}$ & $74(0.207)$ & $137(0.202)$ & 0.869 & NS & 0.974 & $0.709-1.337$ \\
\hline & & $\mathrm{C}$ & $387(0.541)$ & $772(0.570)$ & 0.196 & NS & 1.128 & $0.940-1.353$ \\
\hline & & $\mathrm{T}$ & $329(0.459)$ & $582(0.430)$ & & & 0.887 & $0.739-1.064$ \\
\hline
\end{tabular}

should be greater than 0.05 . According to these criteria, 87 CpG-SNPs were included. However, after these CpGSNPs were subjected to the aforementioned criteria, only 7 CpG-SNPs (rs10454134, rs176249, rs3808620, rs10176517, rs11247118, rs9461624, and rs10492166) were included in the study. Therefore, a total of $10 \mathrm{CpG}-\mathrm{SNPs}$ were included in this study (Table 3 ).

3.3. Genotyping of the CpG-SNPs in BD. Nine CpG-SNPs (rs10454134, rs176249, rs3808620, rs10176517, rs11247118, rs78016579, rs9461624, rs10492166, and rs6507921) were genotyped successfully in $370 \mathrm{BD}$ patients and 704 controls. One CpG-SNP, rs34929465, was excluded, since it could not be analyzed successfully. Seven SNPs found in the healthy controls met the Hardy-Weinberg equilibrium and two SNPs (rs11247118, rs78016579), that deviated from the Hardy-Weinberg equilibrium ( $p$-value<0.05), were excluded. Uncorrected $\mathrm{p}$ values only showed a significant association of rs10454134 with BD (Table 4). A higher frequency of both the rs10454134 AG genotypes $(p=0.008$, 
TABLE 5: Genotype and allele frequencies of rs10454134 in BD and healthy controls (first and second stage cohorts combined).

\begin{tabular}{lcccccccc}
\hline Target_ID & CpG-SNP & Genotype & BD n (\%) & Controls n (\%) & P value & Pc value & OR & 95\%CI \\
\hline cg10181180 & rs10454134 & total sample & 530 & 1034 & & & & \\
& & AA & $126(0.238)$ & $254(0.246)$ & 0.730 & NS & 0.958 & $0.750-1.224$ \\
& & AG & $279(0.528)$ & $485(0.469)$ & 0.032 & NS & 1.258 & $1.020-1.552$ \\
& & GG & $125(0.234)$ & $295(0.285)$ & 0.037 & NS & 0.773 & $0.607-0.985$ \\
& & A & $532(0.502)$ & $993(0.480)$ & 0.250 & NS & 1.091 & $0.941-1.265$ \\
& & G & $528(0.498)$ & $1075(0.520)$ & 0.250 & & 0.917 & $0.791-1.063$ \\
\hline
\end{tabular}

$\mathrm{OR}=1.413,95 \% \mathrm{CI}=1.094-1.826)$ and a lower GG genotype frequency $(\mathrm{p}=0.003, \mathrm{OR}=0.630,95 \% \mathrm{CI}=0.465-0.854)$ were found in $\mathrm{BD}$ patients compared to the controls. After correcting for multiple comparisons, these associations lost statistical significance. To further confirm the trend observed for the CpG-SNP, rs10454134, 172 additional independent BD patients and 330 healthy individuals were recruited. This second stage study also failed to demonstrate an association between this CpG-SNP and BD, even after combining both cohorts (Table 5). Stratified analyses were performed to investigate whether these CpG-SNPs might show an association with the primary clinical features. We chose genital ulcers since this feature has a frequency of approximately $50 \%$ in our BD cohort. After Bonferroni correction, no association was observed after stratification by genital ulcers.

\section{Discussion}

In this study, we failed to find an association between functional CpG-SNPs and BD. Functional CpG-SNPs were selected from data obtained in a previous study, whereby we identified various $\mathrm{CpG}$ sites with a different methylation status in BD patients [19]. The study presented here expanded these findings and investigated whether genetic polymorphisms in these sites might affect predisposition to BD. The fact that no association could be detected suggests that methylation of these sites may not be dependent on genetic variation of these sites themselves but may be regulated by other mechanisms. Further studies are needed to elucidate the exact mechanisms involved.

$\mathrm{BD}$ is considered an autoimmune or autoinflammatory disorder, characterized by chronic and recurrent episodes of posterior or panuveitis. It is generally thought that $\mathrm{BD}$ is caused by the combination of genetic variants and environmental factors. Accumulated evidences in previous studied have unfolded epigenetic control of IL6, IL10, SOCS1, IRF8, GATA3 and TGF- $\beta$ expression participates in the pathogenesis of $\mathrm{BD}$ [8-12]. The introduction or removal of $\mathrm{CpG}$ dinucleotides (possible sites of DNA methylation associated with the environment [6]) has been suggested as a potential mechanism through which SNPs can influence gene transcription and expression via epigenetics [26-29]. Many studies have reported that CpG-SNPs are associated with different diseases, such as type 2 diabetes, breast cancer, coronary heart disease, and psychosis which show a clear interaction between genetic (SNPs) and epigenetic (DNA methylation) regulation [15-18]. The role of SNPs located in CpG sites in autoimmune disease has not been widely addressed and we therefore expanded our earlier studies in this area. We chose $\mathrm{BD}$, since this is a uveitis entity that is commonly observed in China allowing sufficiently large sample sizes to achieve adequate statistical power and allow meaningful conclusions. Identification of genetic variants may lead to novel therapies even in the absence of direct knowledge of the pathogenetic mechanisms involved.

A previous study, where a CpG-SNP MWAS (methylomewide association studies) was performed, showed that CpGSNP rs3796293 reached methylome wide significance in psychosis [18]. Rs3796293 is located in the gene encoding for interleukin 1 receptor accessory protein and supports the role of local inflammation in the pathogenesis of psychosis. In a genome wide association study (GWAS), rs3796293 was not found to be associated with the disease, suggesting that methylation of rs3796293 may not be the directly related to genetic variation of this site [18]. This result is consistent with our study in that genetic variation of significant methylation loci in BD did not show an effect on disease risk.

This study has several limitations. As SNPs were only selected if they satisfied the defined criteria, other unknown functional SNPs in CpG loci with a potential association with $\mathrm{BD}$ may have been missed and need to be investigated in future studies. Despite the fact that we had a large cohort of patients, it is possible that a weak but significant association might have been missed. The gene frequency of the SNPs analyzed in our study has only been tested in normal populations and no reference is available in clinical disease. It should also be noted that methylation changes at certain loci may be the result of the disease rather than its cause.

\section{Conclusion}

In conclusion, this study did not show an association between $\mathrm{BD}$ and $\mathrm{CpG}-\mathrm{SNPs}$ in sites that have an aberrant methylation status.

\section{Data Availability}

All data generated or analyzed during this study are included in this published article.

\section{Conflicts of Interest}

The authors declare that they have no conflicts of interest. 


\section{Acknowledgments}

Thanks to all donors enrolled in the present study. This work was supported by the National Key R\&D Program of China (2016YFC0904000), Natural Science Foundation Major International (Regional) Joint Research Project (81720108009), Chongqing Key Laboratory of Ophthalmology (CSTC, 2008CA5003), Chongqing Science \& Technology Platform and Base Construction Program (cstc2014ptsy10002), and Natural Science Foundation Project of Chongqing (cstc2017shmsA130073).

\section{References}

[1] P. Yang, W. Fang, Q. Meng et al., "Clinical features of chinese patients with Behcet's disease," Ophthalmology, vol. 115, no. 2, pp. 312-318, e314, 2008.

[2] S. Hou, A. Kijlstra, and P. Yang, "Molecular genetic advances in uveitis," Progress in Molecular Biology and Translational Science, vol. 134, pp. 283-298, 2015.

[3] L. Du, A. Kijlstra, and P. Yang, "Immune response genes in uveitis," Ocular Immunology and Inflammation, vol. 17, no. 4, pp. 249-256, 2009.

[4] Y. Huang, H. Yu, Q. Cao et al., "The association of chemokine gene polymorphisms with vkh and behcet's disease in a chinese han population," BioMed Research International, vol. 2017, Article ID 1274960, 8 pages, 2017.

[5] J. Mill and B. T. Heijmans, "From promises to practical strategies in epigenetic epidemiology," Nature Reviews Genetics, vol. 14, no. 8, pp. 585-594, 2013.

[6] R. Feil and M. F. Fraga, "Epigenetics and the environment: emerging patterns and implications," Nature Reviews Genetics, vol. 13, no. 2, pp. 97-109, 2012.

[7] T. Hughes, F. Ture-Ozdemir, F. Alibaz-Oner, P. Coit, H. Direskeneli, and A. H. Sawalha, "Epigenome-wide scan identifies a treatment-responsive pattern of altered dna methylation among cytoskeletal remodeling genes in monocytes and CD4+ T cells from patients with behçet's disease," Arthritis \& Rheumatology, vol. 66, no. 6, pp. 1648-1658, 2014.

[8] S. Alipour, E. Sakhinia, A. Khabbazi et al., "Methylation status of interleukin-6 gene promoter in patients with behcet's disease," Reumatología Clínica, 2018.

[9] A. Abdi, A. Khabazi, E. Sakhinia, S. Alipour, M. Talei, and Z. Babaloo, "Evaluation of SOCS1 methylation in patients with Behcet's disease," Immunology Letters, vol. 203, pp. 15-20, 2018.

[10] S. Alipour, M. Nouri, A. Khabbazi et al., "Hypermethylation of IL-10 gene is responsible for its low mRNA expression in Behcet's disease," Journal of Cellular Biochemistry, vol. 119, no. 8, pp. 6614-6622, 2018.

[11] Y. Qiu, Y. Zhu, H. Yu et al., “Ocular Behcet's disease is associated with aberrant methylation of interferon regulatory factor 8 (IRF8) in monocyte-derived dendritic cells," Oncotarget, vol. 8, no. 31, pp. 51277-51287, 2017.

[12] Y. Zhu, Y. Qiu, H. Yu et al., "Aberrant DNA methylation of GATA binding protein 3 (GATA3), interleukin-4 (IL-4), and transforming growth factor- $\beta$ (TGF- $\beta$ ) promoters in Behcet's disease," Oncotarget, vol. 8, no. 38, pp. 64263-64272, 2017.

[13] R. Shoemaker, J. Deng, W. Wang, and K. Zhang, "Allele-specific methylation is prevalent and is contributed by CpG-SNPs in the human genome," Genome Research, vol. 20, no. 7, pp. 883-889, 2010 .
[14] A. Kapoor, F. Agius, and J.-K. Zhu, "Preventing transcriptional gene silencing by active DNA demethylation," FEBS Letters, vol. 579, no. 26, pp. 5889-5898, 2005.

[15] T. A. Dayeh, A. H. Olsson, P. Volkov, P. Almgren, T. Rönn, and C. Ling, "Identification of CpG-SNPs associated with type 2 diabetes and differential DNA methylation in human pancreatic islets," Diabetologia, vol. 56, no. 5, pp. 1036-1046, 2013.

[16] S. Harlid, M. I. L. Ivarsson, S. Butt et al., "A candidate CpG SNP approach identifies a breast cancer associated ESR1-SNP," International Journal of Cancer, vol. 129, no. 7, pp. 1689-1698, 2011.

[17] X. Chen, X. Chen, Y. Xu et al., "Association of six CpG-SNPs in the inflammation-related genes with coronary heart disease," Human Genomics, vol. 10, no. 2, p. 21, 2016.

[18] E. J. C. G. Van Den Oord, S. L. Clark, L. Y. Xie et al., "A whole methylome CpG-SNP association study of psychosis in blood and brain tissue," Schizophrenia Bulletin, vol. 42, no. 4, pp. 10181026, 2016.

[19] H. Yu, L. Du, S. Yi et al., "Epigenome-wide association study identifies Behcet's disease-associated methylation loci in Han Chinese," Rheumatology, 2019.

[20] "Criteria for diagnosis of Behcet's disease. International Study Group for Behcet's Disease," The Lancet, vol. 335, no. 8697, pp. 1078-1080, 1990.

[21] Y. Zou, Q. Li, L. Jiang et al., "DNA hypermethylation of CREB3L1 and Bcl-2 associated with the mitochondrialmediated apoptosis via PI3K/Akt pathway in human BEAS-2B cells exposure to silica nanoparticles," PLoS ONE, vol. 11, no. 6, Article ID e0158475, 2016.

[22] J. Liu and K. D. Siegmund, "An evaluation of processing methods for HumanMethylation450 BeadChip data," BMC Genomics, vol. 17, article 469, 2016.

[23] L. D. Ward and M. Kellis, "HaploReg: a resource for exploring chromatin states, conservation, and regulatory motif alterations within sets of genetically linked variants," Nucleic Acids Research, vol. 40, no. 1, pp. D930-D934, 2012.

[24] L. D. Ward and M. Kellis, "HaploReg v4: systematic mining of putative causal variants, cell types, regulators and target genes for human complex traits and disease," Nucleic Acids Research, vol. 44, no. 1, pp. D877-D881, 2016.

[25] P. A. Jones, "Functions of DNA methylation: islands, start sites, gene bodies and beyond," Nature Reviews Genetics, vol. 13, no. 7, pp. 484-492, 2012.

[26] A. Bird, "The dinucleotide CG as a genomic signalling module," Journal of Molecular Biology, vol. 409, no. 1, pp. 47-53, 2011.

[27] C. Hsieh, "Dependence of transcriptional repression on $\mathrm{CpG}$ methylation density," Molecular and Cellular Biology, vol. 14, no. 8, pp. 5487-5494, 1994.

[28] G. C. Prendergast and E. B. Ziff, "Methylation-sensitive sequence-specific DNA binding by the c-Myc basic region," Science, vol. 251, no. 4990, pp. 186-189, 1991.

[29] A. M. Deaton and A. Bird, "CpG islands and the regulation of transcription," Genes \& Development, vol. 25, no. 10, pp. 10101022, 2011. 


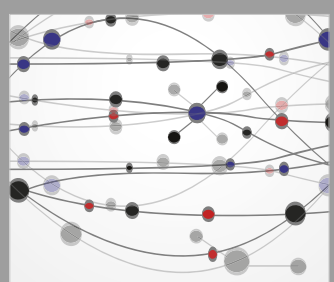

The Scientific World Journal
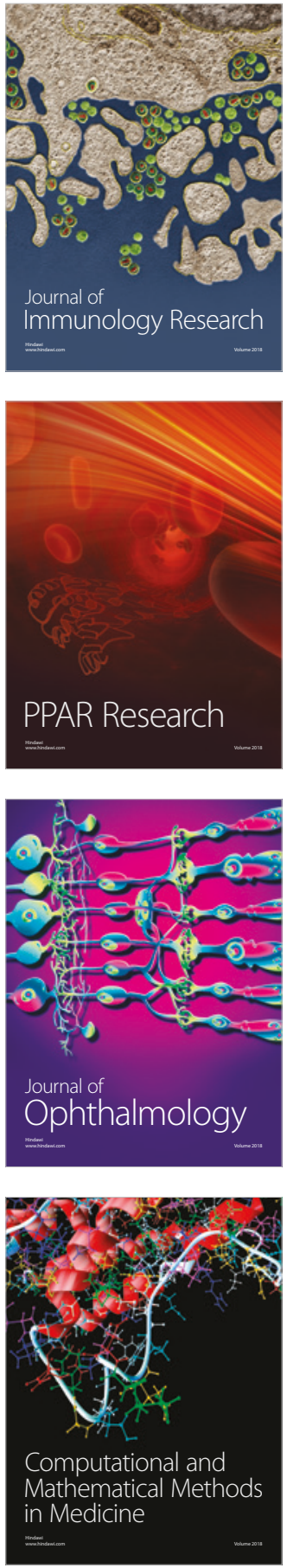

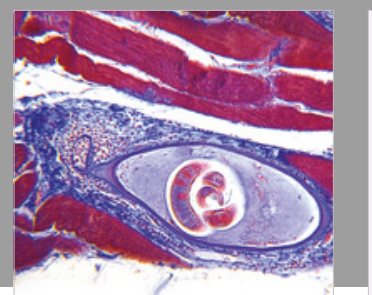

Gastroenterology Research and Practice

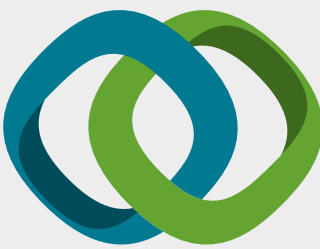

\section{Hindawi}

Submit your manuscripts at

www.hindawi.com
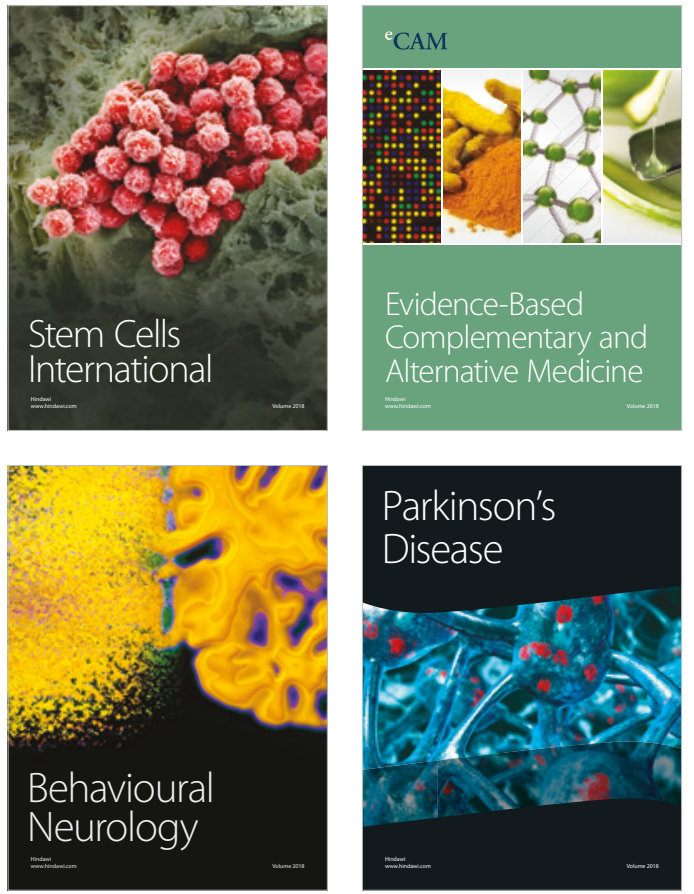

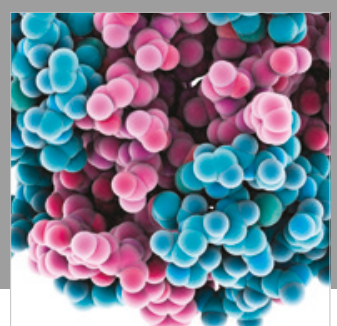

ournal of

Diabetes Research

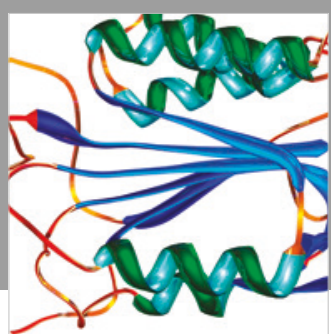

Disease Markers
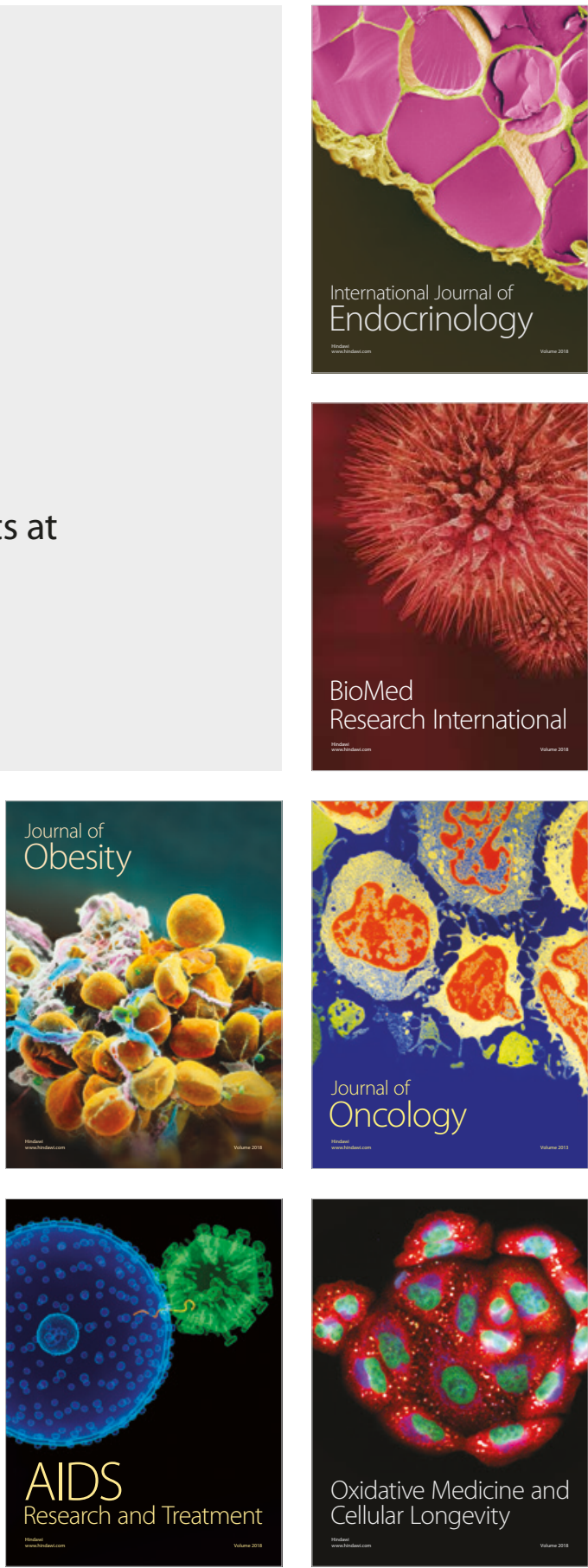